\title{
DJ-1 isoforms in whole blood as potential biomarkers of Parkinson disease
}

SUBJECT AREAS:

PARKINSON'S DISEASE

DIAGNOSTIC MARKERS

MASS SPECTROMETRY

PROTEOMICS

Received

17 July 2012

Accepted

26 October 2012

Published

11 December 2012

Correspondence and requests for materials should be addressed to J.Z. (zhangi@uw.edu)

* These authors contributed equally to this work.
Xiangmin Lin ${ }^{1,2 *}$, Travis J. Cook ${ }^{3 *}$, Cyrus P. Zabetian ${ }^{4,5,6}$, James B. Leverenz ${ }^{4,5,6,7}$, Elaine R. Peskind 7,8 , Shu-Ching Hu ${ }^{6}$, Kevin C. Cain 9 , Catherine Pan ${ }^{1}$, John Scott Edgar ${ }^{10}$, David R. Goodlett ${ }^{10}$, Brad A. Racette ${ }^{11}$, Harvey Checkoway ${ }^{3}$, Thomas J. Montine ${ }^{1}$, Min Shi' \& Jing Zhang'

'Department of Pathology, University of Washington School of Medicine, Seattle, WA, USA, ${ }^{2}$ School of Life Sciences, Fujian Agricultural and Forestry University, Fuzhou 35002, People's Republic of China, ${ }^{3}$ Department of Environmental \& Occupational

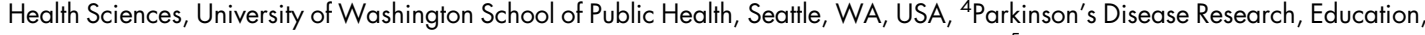
and Clinical Center, Veterans Affairs Puget Sound Health Care System, Seattle, WA, USA, ${ }^{5}$ Geriatric Research, Education, and Clinical Center, Veterans Affairs Puget Sound Health Care System, Seattle, WA, USA, ${ }^{\circ}$ Department of Neurology, University of Washington School of Medicine, Seattle, WA, USA, ${ }^{7}$ Mental Illness Research, Education, and Clinical Center, Veterans Affairs Puget Sound Health Care System, Seattle, WA, USA, ${ }^{8}$ Department of Psychiatry and Behavioral Sciences, University of Washington School of Medicine, Seattle, WA, USA, 'Department of Biostatistics, University of Washington School of Public Health, Seattle, WA, USA, ${ }^{10}$ Department of Medicinal Chemistry, University of Washington School of Pharmacy, Seattle, WA, USA, " 1 Department of Neurology, Washington University School of Medicine, St. Louis, MO, USA.

DJ-1 is a multifunctional protein that plays an important role in oxidative stress, cell death, and synucleinopathies, including Parkinson disease. Previous studies have demonstrated that total DJ-1 levels decrease in the cerebrospinal fluid, but do not change significantly in human plasma from patients with Parkinson disease when compared with controls. In this study, we measured total DJ-1 and its isoforms in whole blood of patients with Parkinson disease at various stages, Alzheimer disease, and healthy controls to identify potential peripheral biomarkers of PD. In an initial discovery study of 119 subjects, $7 \mathrm{DJ}-1$ isoforms were reliably detected, and blood levels of those with 4-hydroxy-2-nonenal modifications were discovered to be altered in late-stage Parkinson disease. This result was further confirmed in a validation study of another 114 participants, suggesting that, unlike total DJ-1 levels, post-translationally modified isoforms of DJ-1 from whole blood are candidate biomarkers of late-stage Parkinson disease.
P arkinson disease (PD) is a chronic, progressive movement disorder that is the second most common neurodegenerative disease after Alzheimer disease $(\mathrm{AD})^{1}$. Recent epidemiologic data estimates overall PD prevalence to be approximately $1.6 \%$ among people 65 years of age or older, although prevalence rates among some combinations of ethnicity, gender, and geographic location are observed to be much higher in the elderly population ${ }^{2}$. Although PD was first described nearly 200 years ago, its pathogenic mechanisms remain unclear. Currently, PD diagnosis is essentially based upon patient medical history, along with observation of the cardinal motor indicators of the illness and drug treatment effects ${ }^{3}$. However, there is an appreciable misdiagnosis rate $^{4}$ and there is no established way of objectively monitoring disease progression. Thus, biomarkers are sorely needed to aid in both the diagnosis and in monitoring the severity/progression of PD. To date, one of the most extensively tested markers in human cerebrospinal fluid (CSF) is DJ- $1^{5}$, in which mutation causes autosomal recessive $\mathrm{PD}^{6}$ and is also believed to be important in idiopathic $\mathrm{PD}$ particularly due to its antioxidative activity and associated neuroprotection ${ }^{7}$. However, accessing CSF is not a trivial task in a regular clinical setting, let alone in a screening assay that can be used for early-stage diagnosis.

In contrast to CSF, blood is a much more readily accessible source for clinical application and is widely used for measuring biomarkers in a variety of illnesses ${ }^{8-11}$. It has been reported that plasma DJ-1 levels do not significantly differ between controls and patients with PD or $\mathrm{AD}^{12}$. However, $>95 \%$ of blood-based DJ-1 is contained within red blood cells $(\mathrm{RBCs})^{12}$ and the levels of total DJ-1 and its isoforms, including those with post-translational modifications (PTMs), are still unknown in whole blood samples of PD patients and controls. In this study, we began by assessing the total amount of DJ-1 and levels of its isoforms among PD, AD, and control blood using a combination of one- or two-dimensional gel electrophoresis (2-DE) with quantitative western blot analysis to provide clues for the development of a PD biomarker screen in human blood samples. DJ-1 isoforms with PTMs were evaluated with mass spectrometry (MS), and novel isoforms discovered to be modified with 4-hydroxy-2nonenal (4-HNE) were further investigated as potential biomarkers of PD diagnosis as well as disease severity. 


\section{Results}

Total DJ-1 amount in PD vs. control blood samples. Since a majority of total DJ-1 is attributable to RBCs, we evaluated the potential use of DJ-1 in whole blood as a biomarker of PD. To accomplish this goal, a total of 119 participants in the discovery set were separated into 5 diagnostic sets and classified as controls, AD patients, and early-, middle- or late-stage PD patients according to Unified Parkinson Disease Rating Scale (UPDRS) score (Table 1). Total DJ-1 was measured and signal densities were analyzed as compared to controls (Fig. 1). Although there was a tendency of total DJ-1 levels to increase slightly with higher UPDRS score, statistical significance was not achieved.

Identification of DJ-1 isoforms in whole blood samples. Next, we asked whether isoforms of DJ-1 could be used as biomarkers of PD diagnosis or PD severity. DJ-1 is a low abundance protein and comigration of proteins is a well-known phenomenon in twodimensional electrophoresis (2-DE) analysis ${ }^{20}$. Thus, it is difficult to detect DJ-1 unequivocally by MS in whole blood with 2-DE separation when unfractionated samples are used. For this reason, total DJ-1 (MW 19.9 kDa) was enriched using differential solubilization (DS) methods before being submitted to MS for identification. Multiple isoforms (2-DE spots) of DJ-1 at approximately $20 \mathrm{kDa}$ and a $\mathrm{pH}$ range of about $5-7$ were identified by silver staining and western blotting using an anti-DJ-1 antibody (Fig. 2A, Inset; and Supplemental Fig. 1). We confirmed 7 spots to be isoforms of DJ-1 by LCLTQ-Obitrap MS (Table 3). Representative spectra from two matched peptide masses $(\mathrm{MH}+1675.8035$ and $\mathrm{MH}+1259.8259)$ are shown in Fig. 2. Although the nature of each DJ-1 isoform remains to be characterized, these isoelectric variants are believed to result from PTMs that alter the intrinsic charge of the protein. Indeed, 4-HNE modifications at Lys-32 were identified in isoforms 4 and $6(\mathrm{MH}+1212.7881$, Fig. 3A) and at Lys-62 in isoform $6(\mathrm{MH}+$ 687.8409 , Fig. 3B) in at least two independent experiments with mass errors as low as $0.278 \mathrm{ppm}$. To further confirm these modifications, western blotting using an antibody against HNE was performed after DS enrichment and 2-DE (Fig. 3A, Inset), and then the HNEpositive spots were confirmed to be DJ-1 after re-probing with the anti-DJ-1 antibody. Similar results were obtained when proteins were enriched with IP before 2-DE and western blotting (Supplemental Fig. 2A and 2B). Additional modifications identified by LTQ-Oribitrap MS included cysteic acid modification of isoform 4 at Cys-106 and phosphorylation of isoforms 4, 5 and 6 at Thr-19 (Fig. 3C, Table 3). The phosphorylated DJ-1 isoform was also further confirmed by IP of a much larger volume of blood $(4 \mathrm{~mL})$ before 2$\mathrm{DE}$ and western blotting using an anti-phospho-Thr antibody
(Supplemental Fig. 2C and 2D). Besides these PTMs, methionine sulfoxide modifications were also found at Met-17 and Met-26 in different spots.

DJ-1 isoforms in PD vs. control blood samples. To investigate the characteristics of DJ-1 isoforms in the diagnosis of PD or their correlation with PD severity, the same comparison groups described for total DJ-1 analysis were evaluated. At least 5 isoforms of DJ-1, named as isoforms 2 through 6 , were visualized in each group after western blotting of the pooled whole blood samples (Fig. 4A). Of note, a quantitative comparison of DJ-1 isoforms was performed in the samples without pre-enrichment. Isoforms were named in reference to those shown in Fig. 2. The expression level of DJ-1 isoforms vs. total DJ-1 level for each group was measured and quantified as volume percentage (Fig. 4B). As compared to controls, the results showed that isoform 2 was significantly decreased in $\mathrm{AD}$ and all stages of $\mathrm{PD}$. Conversely, isoform 6 was significantly increased in AD and slightly increased in all stages of PD as compared to controls. However, none of the isoforms showed clear correlation with PD severity. No significant changes were observed among isoforms 3, 4, or 5 (Fig. 4C and 4D).

Discovery of HNE-modified DJ-1 isoforms in PD vs. control blood samples. Among four major PTMs identified by MS (methionine oxidation, which could be an ex vivo artifact, cysteine oxidation, phosphorylation, and HNE adduction), HNE adduction was the only PTM that could be followed with an antibody specific and sensitive enough in our platform. The specificity of the antibody against reduced HNE adducts ${ }^{15}$ was confirmed by dot blotting of HNE-modified BSA and DJ-1 and 2-DE western blotting of whole blood samples with or without reduction by $\mathrm{NaBH}_{4}$, using the antiHNE antibody or pre-immunity serum (Supplemental Fig. 3). To further investigate HNE-altered DJ-1 among PD, AD, and control samples, the level of HNE-modified DJ-1 fraction in each DJ-1 isoform or total HNE-modified DJ-1 was quantified. The HNEmodified DJ-1 isoform 4 as a fraction of total isoform 4 level (Fig. 5B) or as a fraction of total HNE-modified DJ-1 (isoform 4 +6 ) level (Fig. 5C) tended to decrease as a function of PD severity, with statistical significance achieved in patients with more advanced diseases. In contrast, although the ratio of the expression of HNEmodified isoform 6 over total isoform level did not change significantly for any group (Fig. 5D), the ratio of isoform 6 as a fraction of total HNE-modified forms (Fig. 5E) was increased in late-stage PD patients compared to early-stage $\mathrm{PD}$ patients, $\mathrm{AD}$ patients, and controls.

Validation of HNE-modified DJ-1 isoforms in PD vs. control blood samples. To further confirm the HNE-modified DJ-1 result

\begin{tabular}{|lccccc|}
\hline $\begin{array}{l}\text { Table 1 | Blood group data of patients and controls for discovery } \\
\text { Sample }\end{array}$ & Group & Cases & Age (Mean \pm SD) & M:F & UPDRS (Mean \pm SD) \\
\hline Control & 1 & 10 & $64.2 \pm 8.417$ & $6: 4$ \\
& 2 & 10 & $64.2 \pm 9.760$ & $6: 4$ \\
AD & 3 & 10 & $64.3 \pm 7.379$ & $6: 4$ & $2: 2$ \\
& 1 & 4 & $67.8 \pm 7.823$ & $3: 2$ & $2: 3$ \\
PD (UPDRS $<15)$ & 2 & 5 & $67.8 \pm 8.955$ & $2: 3$ & $12.0 \pm 5.339$ \\
& 3 & 5 & $67.4 \pm 6.768$ & $2: 3$ & $10.1 \pm 3.232$ \\
PD (UPDRS 15-30) & 1 & 5 & $63.6 \pm 9.236$ & $6: 3$ & $20.7 \pm 4.714$ \\
& 2 & 5 & $62.6 \pm 11.104$ & $6: 4$ & $21.5 \pm 3.154$ \\
PD (UPDRS $>30)$ & 3 & 5 & $63.2 \pm 11.520$ & $6: 4$ & $21.6 \pm 4.122$ \\
& 1 & 10 & $63.8 \pm 7.146$ & 63.8 & $47.6 \pm 9.095$ \\
Total & 2 & 10 & $63.8 \pm 7.254$ & $6: 4$ & $47.2 \pm 8.417$ \\
\end{tabular}




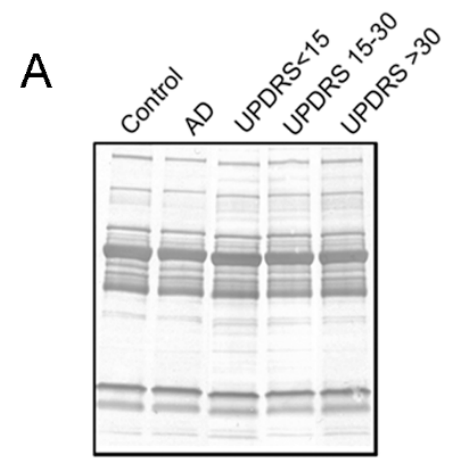

B
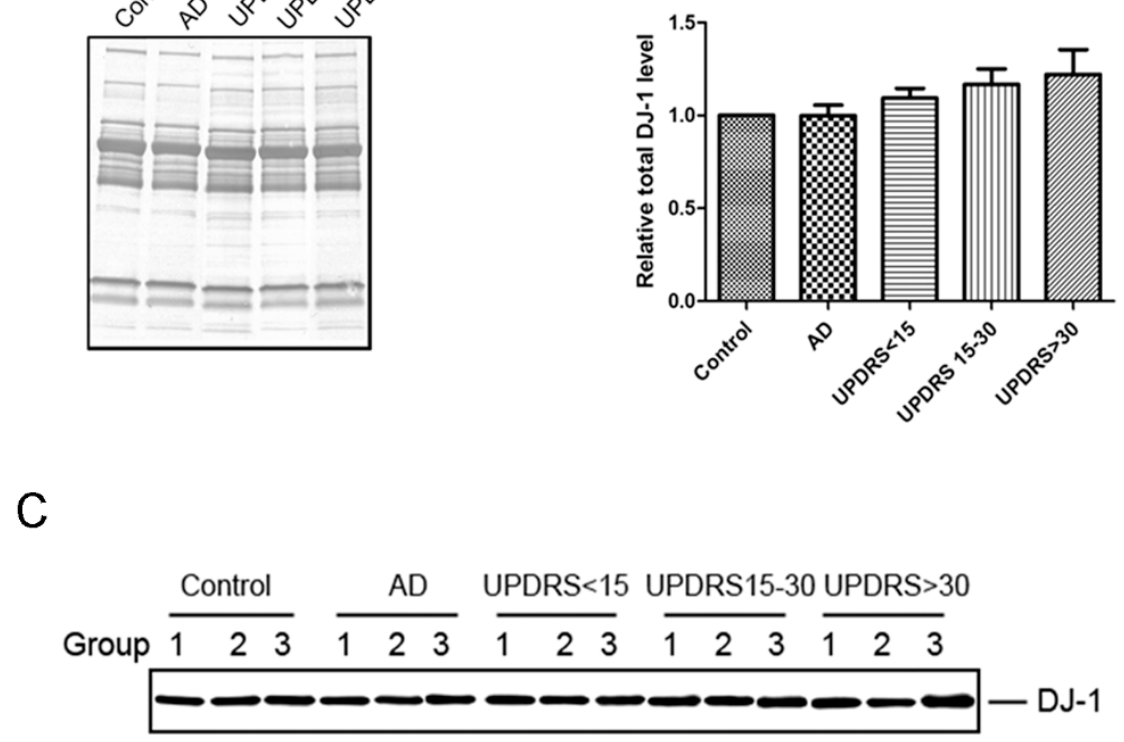

Figure $1 \mid$ Total DJ-1 levels in whole blood were not significantly different among patients with PD at various stages, AD, and controls. Pooled whole blood samples from controls, patients with Alzheimer disease (AD) and patients with Parkinson disease (PD) at various stages (UPDRS motor score $<15$, 15-30, and >30) were analyzed by 1-DE western blotting. The samples were pooled according to Tablel (five comparison groups with each pooled into three sub-groups). (A) Coomassie R-350 staining of the membrane indicated equal loading among the five samples. This is a representative result from one sub-group of three independent experiments. (B) Quantitative western blotting against DJ-1 of the 5 groups indicated total DJ-1 does not significantly change with disease status. The adjusted DJ-1 band volumes were normalized to the control values in each independent experiment/gel. A representative gel is shown in $(\mathrm{C})$.

in PD patients, a validation study of 114 subjects including controls $(\mathrm{n}=30)$ and patients with early- $(\mathrm{n}=24)$, intermediate- $(\mathrm{n}=30)$, and late-stage PD $(n=30)$ was performed. As indicated in Fig. 5, the general tendencies of HNE-modified DJ-1 isoforms 4 and 6 are consistent in comparing the discovery and validation cohorts. HNE-modified isoform 4 as a fraction of both isoform 4 level (Fig. 5B) and total HNE-modified DJ-1 (Fig. 5C) tended to decrease in both cohorts. Importantly, statistical significance was achieved in both cohorts when comparing late-stage PD with controls or early-stage PD. This isoform also exhibited a "progressive" characteristic, with decreasing expression of the HNE-modified form of the isoform associated with increasing disease severity. Additionally, while HNE-modified isoform 6 exhibited no correlation with disease as a fraction of total isoform 6 level (Fig. 5D), it was found to be increased as a fraction of total HNE-modified DJ-1 when comparing controls and early-stage PD patents to intermediate- and late-stage PD patients (Fig. 5E).

\section{Discussion}

Several interesting findings were made as a result of the present study. First, similar to what we described in plasma, total DJ-1 levels in whole blood were not changed significantly in either AD or PD patients compared with age-matched controls. Second, 7 isoforms of DJ-1 in whole blood were identified, with several PTMs within those isoforms, including phosphorylation and oxidative modifications. Most importantly, we observed significant neurodegenerative disease-related differences in the whole blood levels of two of these isoforms, with HNE adduction, that were unique to PD diagnosis or disease severity.

As discussed earlier, unlike CSF, total DJ-1 in plasma is not an effective marker for differentiating PD patients from controls and is not correlated with PD severity ${ }^{12}$. More than $95 \%$ of blood DJ-1 is contained in $\mathrm{RBCs}^{12}$, and variable in vitro or in vivo hemolytic changes that are beyond investigators' control likely contributed to contradicting reports in the literature regarding its plasma levels ${ }^{12,21-23}$. Our study has the advantage that it was carried out in whole blood, eliminating the possibility of confounding by component contamination. The results of our study, which used quantitative western blotting, are in accordance with the findings that total DJ-1 levels in blood do not change based on neurodegenerative disease status. This observation further indicates that total DJ-1 is not a useful biologic indicator of PD in blood or in blood components.

In contrast to total DJ-1 levels, we found that some isoforms in whole blood samples were significantly different in both PD and AD patients when compared with controls. Specifically, isoform 2 was lower in both $\mathrm{PD}$ and $\mathrm{AD}$ patients, while isoform 6 was higher in both disease groups. However, while these DJ-1 isoforms may have potential as biomarkers of neurodegenerative disease, neither isoform exhibited specificity for either disease nor did these correlate with PD severity. It should be noted that the inability to differentiate $\mathrm{AD}$ and $\mathrm{PD}$ patients based on these isoforms does not necessarily negate the potential clinical utility of these candidate biomarkers, as $\mathrm{AD}$ and $\mathrm{PD}$ are easily distinguishable by clinicians using current diagnostic methods. A more important question to ask in future research is whether DJ-1 isoforms can distinguish PD from other parkinsonian conditions that overlap with PD clinically, such as multiple system atrophy and progressive nuclear palsy. The diagnosis of PD and disease progression monitoring are currently mainly based on the evaluation of clinical symptoms, which is subjective and thus often associated with a considerable error rate. More recently, several neuroimaging techniques may be employed to help clinicians in assessing PD and its progression ${ }^{24}$. However, there are several notable limitations to these techniques, including limited specificity and accountability for compensatory mechanisms. It is therefore vital that biochemical markers such as the ones described here as well as more robust specific ELISA and Luminex assays continue to be developed in an effort to complement the inherent shortcomings of neuroimaging biomarkers. 

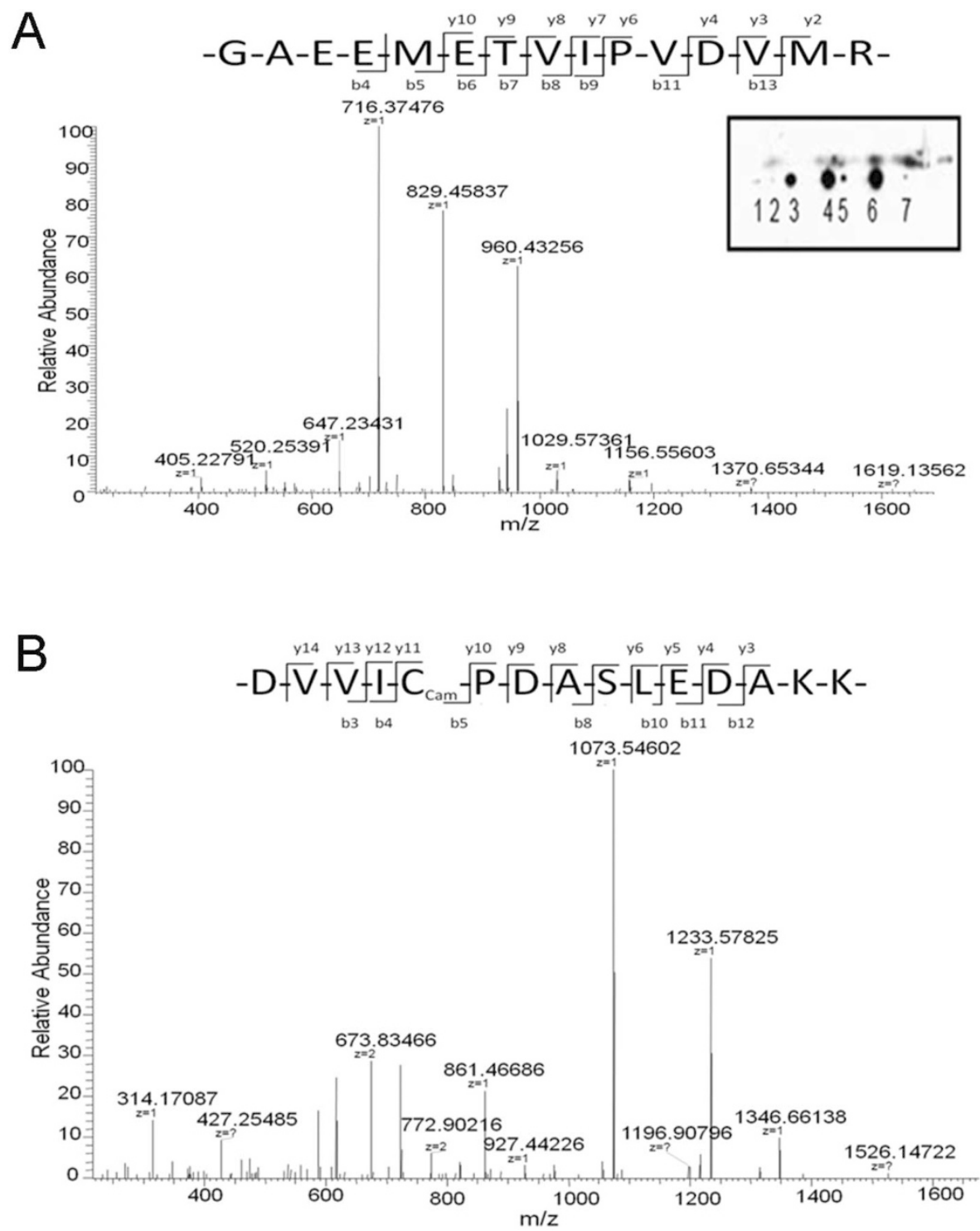

Figure $2 \mid$ DJ-1 isoforms in whole blood were confirmed by MS/MS and western blotting. (A) 2-DE western blotting against DJ-1 identified 7 spots as DJ-1 isoforms (Inset). LTQ-Obitrap MS/MS confirmed the 7 spots to be DJ-1. Shown is a MS2 spectrum of MH+ 1675.8035 indicating a DJ-1 peptide with the sequence GAEEMETVIPVDVMR. (B) MS2 spectrum indicating MH+ 1259.8259 is a DJ-1 peptide with the sequence DVVICPDASLEDAKK.

Another major discovery of our investigation relates to the characterization of various PTMs of DJ-1 with MS and western blotting. Although methionine oxidation can occur ex vivo during sample processing (i.e., its significance is unclear) and phosphorylation has been reported at Tyr- $67^{25}$, both phosphorylation at Thr-19 and HNE adduction of DJ-1 are novel and potentially important to PD pathogenesis in addition to their utility as biomarkers. To this end, it should be stressed that oxidatively modified DJ-1 has been reported to be elevated in brain tissues of both $\mathrm{PD}$ and $\mathrm{AD}$ patients ${ }^{26}$, and oxidized DJ-1 protein has been previously suggested to be a useful biomarker as it has been recently demonstrated that levels of DJ-1 oxidation at Cys-106 are elevated in the RBCs of unmedicated PD patients when compared to both medicated patients and controls ${ }^{27}$. Several investigations clearly suggest that the Cys-106 oxidative modification is critically involved in PD pathogenesis ${ }^{28-31}$. The issue, of course, is whether observations in the CNS are related to peripheral modifications of DJ-1. While this question cannot be answered by the current study alone, cysteine-sulfonic acid modification of Cys-106 in isoform 4 was indeed observed in the current investigation. Importantly, this modification was not observed in the much more abundant isoform 6, suggesting this highly relevant PTM may be specific to a distinct DJ-1 isoform. Additionally, in vivo studies demonstrate that dosing rats with the neurotoxicants 1methyl-4-phenyl-1,2,3,6-tetrahydropyridine or 6-hydroxydopamine results in elevated levels of Cys-106 oxidized DJ-1 in $\mathrm{RBCs}^{32}$, indicating that the modification in blood DJ-1 may not only be a surrogate maker, but also be biologically relevant to $\mathrm{PD}$. It should also be stressed that one of the prevailing schools of thought is that PD is not just a CNS disease as it has a significant peripheral component, including generalized mitochondrial dysfunction in platelets, constipation, and cardiac arrhythmia due to denervation of the autonomic nervous system.

HNE modification of DJ-1, though not reported previously even in the CNS, is clearly consistent with the generally accepted hypothesis that PD patients experience substantially increased levels of oxidative stress, with multiple proteins containing HNE adduction $^{33,34}$. The fact that HNE-modified DJ-1 isoform 4 significantly decreased, while isoform 6 increased, in late-stage PD patients suggests that these modified DJ-1 isoforms could potentially be biomarkers of disease severity in a body fluid that is readily accessible clinically. The on-state UPDRS motor score was selected as an index of PD severity in this study, as it has been utilized effectively to reflect 


\begin{tabular}{|c|c|c|c|c|c|}
\hline Sample & Group & Cases & Age (Mean $\pm S D)$ & $M: F$ & UPDRS (Mean \pm SD) \\
\hline \multirow[t]{2}{*}{ Control } & 1 & 10 & $63.0 \pm 11.431$ & $6: 4$ & \\
\hline & 3 & 10 & $63.6 \pm 8.872$ & $5: 5$ & \\
\hline \multirow[t]{2}{*}{ PD (UPDRS < 15) } & 1 & 8 & $65.5 \pm 7.164$ & $4: 4$ & $10.4 \pm 3.777$ \\
\hline & 2 & 8 & $56.3+8.678$ & $4: 4$ & $11.1 \pm 2.295$ \\
\hline \multirow{2}{*}{ PD (UPDRS 15-30) } & 2 & 10 & $62.1 \pm 4.156$ & $6: 4$ & $22.2 \pm 5.095$ \\
\hline & 3 & 10 & $62.8 \pm 7.323$ & $6: 4$ & $21.6 \pm 4.300$ \\
\hline \multirow[t]{3}{*}{ PD (UPDRS > 30) } & 1 & 10 & $72.1 \pm 8.084$ & $6: 4$ & $46.3 \pm 8.509$ \\
\hline & 2 & 10 & $71.1 \pm 9.692$ & $6: 4$ & $45.4 \pm 9.300$ \\
\hline & 3 & 10 & $71.3 \pm 7.678$ & $6: 4$ & $46.8 \pm 11.708$ \\
\hline Total & & 114 & & & \\
\hline
\end{tabular}

clinical progression of motor impairments in patients with PD; in both the discovery and validation cohorts, patients with higher UPDRS motor scores also tended to have longer duration of disease, another commonly used indicator of the disease severity. However, it should be noted that both indices used to stage PD patients are prone to several limitations that may lead to misclassification bias. For UPDRS motor scores, these include differential benefit from levodopa, physician biases in treatment, subject expectations, and nonmotor signs which contribute substantially to disability. Similarly, disease duration can only partially reflect the disease severity or progression.

The molecular mechanisms underlying quantitative alterations of different DJ-1 isoforms remain to be characterized. One possibility is that HNE-modified DJ-1 (indicative of damaged protein) is targeted for degradation more readily by proteasome or lysosome systems, resulting in a decrease of isoform 4 . Such a phenomenon has been demonstrated in vitro where it has been found that HNE can decrease DJ-1 levels and induce cell death in HT22 cells ${ }^{35}$. However, the process could be much more complicated, given that the opposite trend was observed for isoform 6 . A more likely explanation could be that there is a PD-specific shift of HNE-modification from DJ-1 isoform 4 to 6 as the disease progresses, a hypothesis that needs to be tested in future investigations.

Two HNE-modified peptides were identified with high confidence in this study; for example, we were able to measure the mass of D-VV-I-C-P-D-A-S-L-E-D-A-K (HNE) $_{\text {to }}$ to within a $0.278 \mathrm{ppm}$ mass error compared to theoretical values using an LTQ-Orbitrap. However, the two reported peptides consist of a mis-cleavage (Nflanking sequence: -R-A-G-) and a modified C-terminal (C-flanking residue: $-\mathrm{K}-$ ), respectively. There are a few potential underlying reasons that may result in proteolytic miscleavage and/or modified terminals, including protein structure, enzymatic impurities, and post-tryptic mechanisms such as chemical hydrolysis. The MS data suggest that these two locations represent the strongest candidate sites of HNE-modification. Given that we have also confirmed the existence of HNE modifications in the 2-DE gel spots using western blotting after both the DS and IP enrichments, it is reasonable to conclude that HNE-modified proteins exist in these samples.

It should also be noted that although several novel PTMs were identified in the current investigation, the migration pattern cannot necessarily be explained by the identified PTMs alone. A recent metaanalysis of the DJ-1 2DE literature indicated acidic isoforms of the

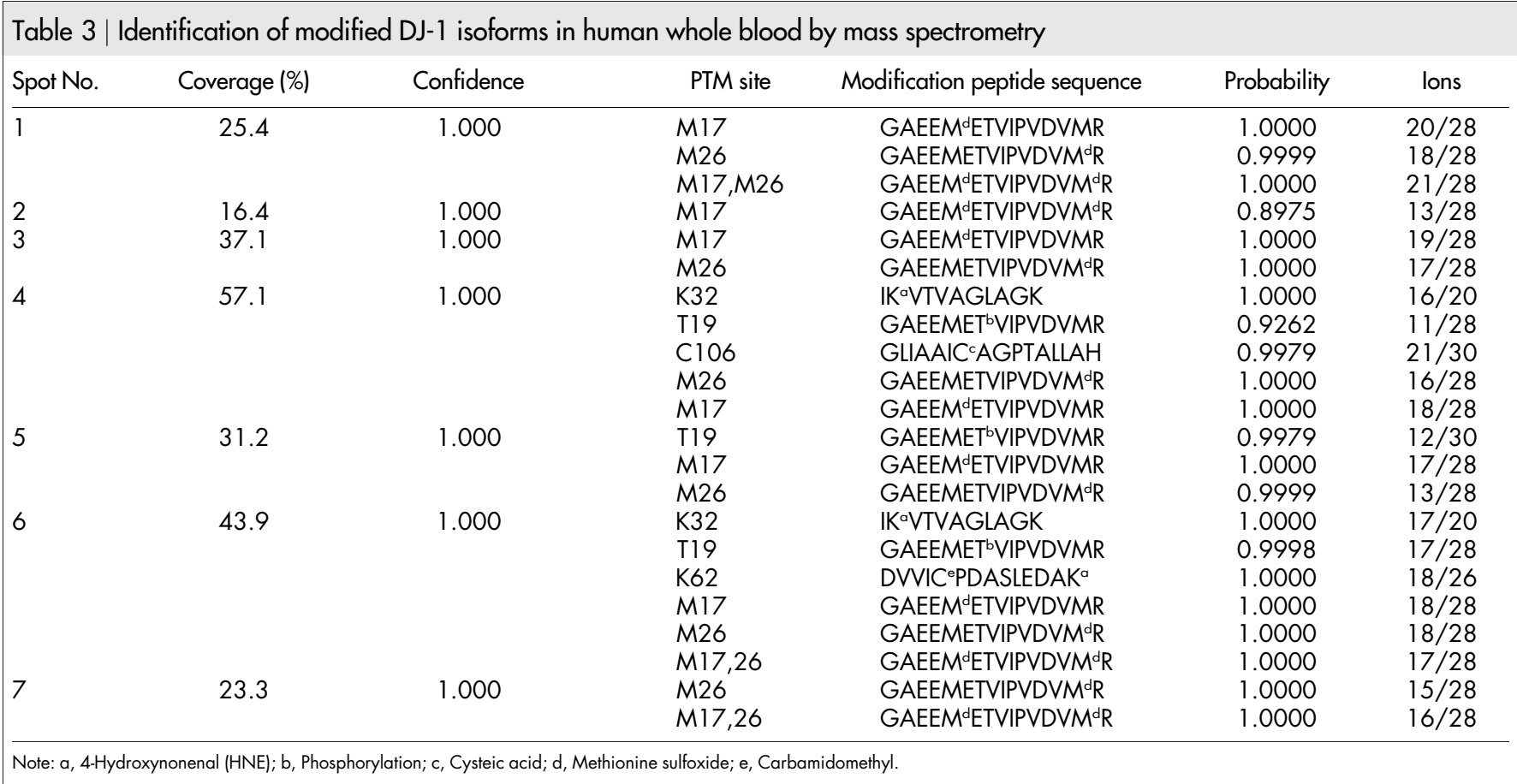



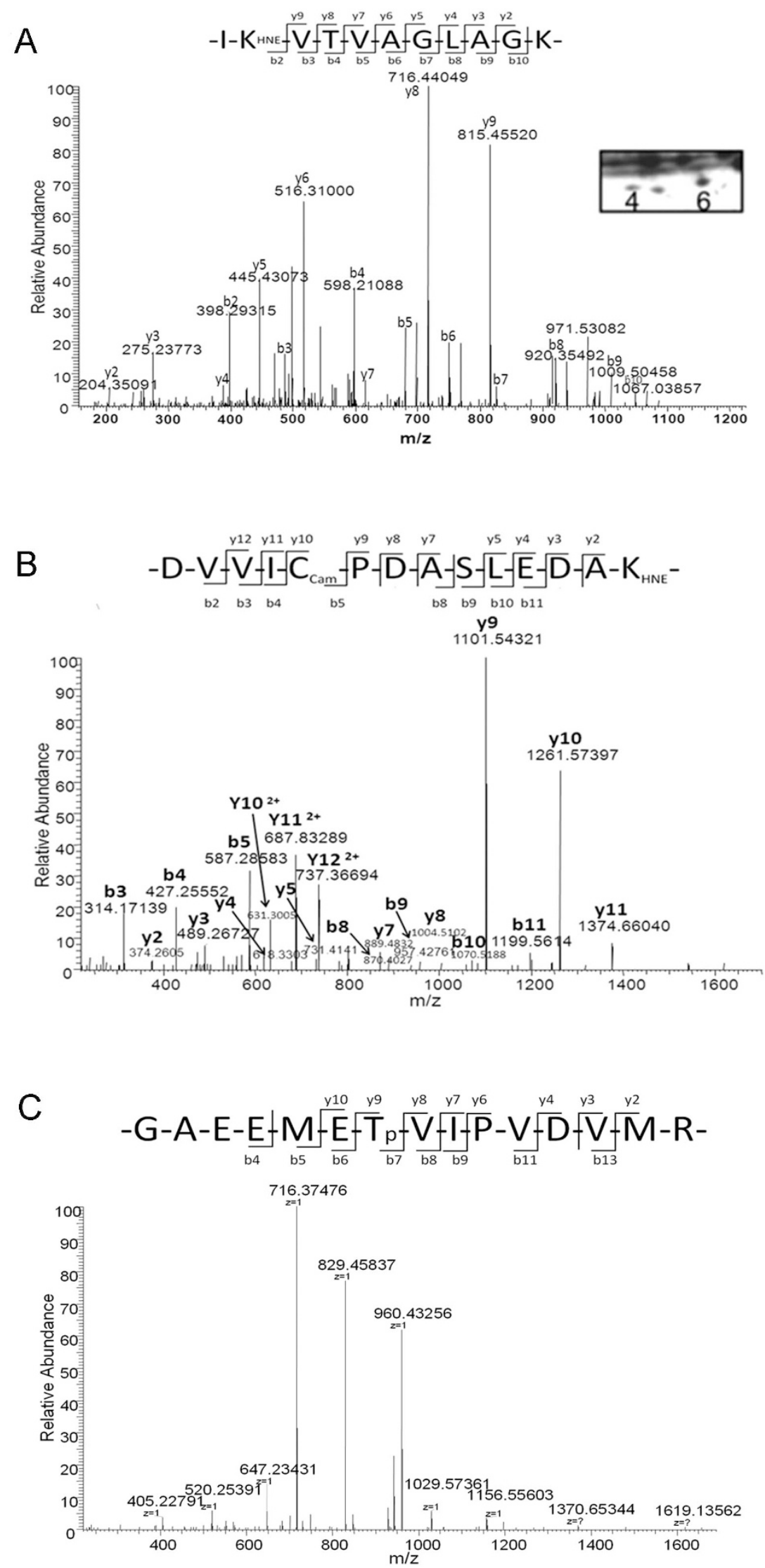

Figure 3 4-HNE and phosphorylation modifications of DJ-1 in whole blood were identified by MS/MS. (A) Western blotting confirmed HNE modification of DJ-1 isoforms. The right-hand inset shows HNE western blotting before stripping of the DJ-1 membrane. Left, LTQ-Obitrap MS/MS spectrum indicated MH+ 1212.7881 is a DJ-1 HNE-modified peptide in spots 4 and 6 at Lys-32. (B) MS2 spectrum indicated MH+ 687.8409 is a DJ-1 HNE-modified peptide in spot 6 at Lys-62. (C) MS2 spectrum indicated MH+ 1771.7635 is a DJ-1 phosphorylation-modified peptide in isoforms 4, 5 and 6 at Thr- 19 . 
A

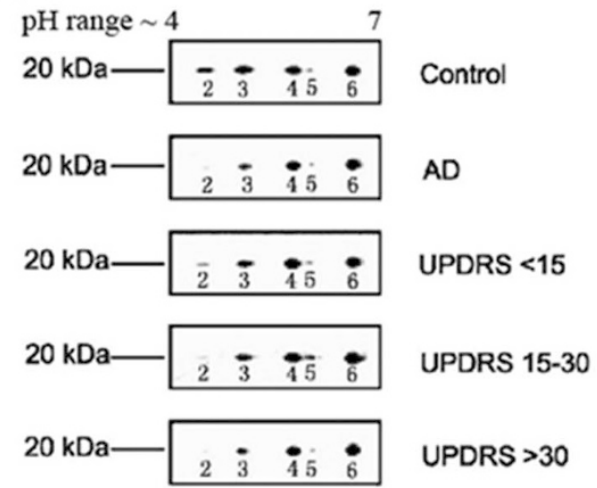

C

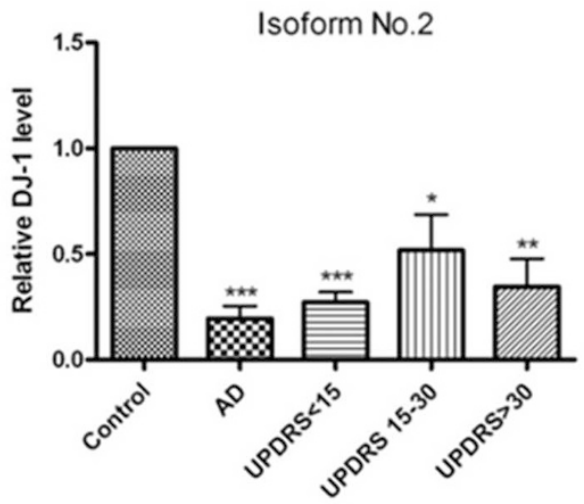

B

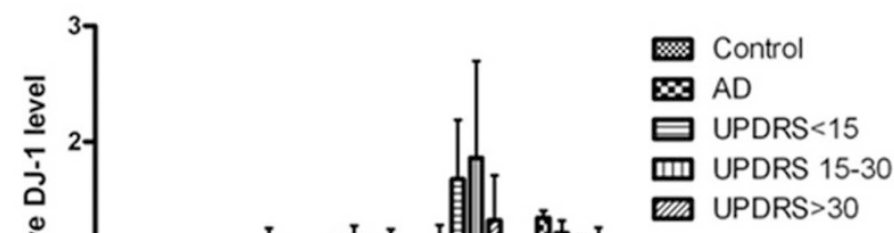

D

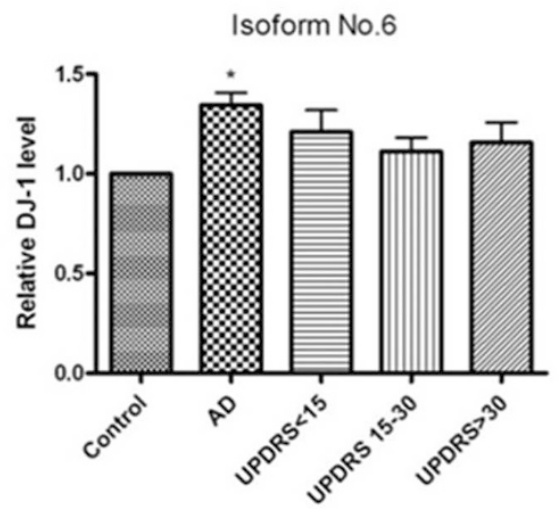

Figure $4 \mid$ The expression of DJ-1 isoforms was significantly different among control and disease whole blood samples. (A) 2-DE western blotting of DJ-1 isoforms in different pooled whole blood samples (Table 1); this is a representative result from one of the three sub-groups of three independent experiments. (B) Quantification of 2-DE western blotting against DJ-1; the adjusted DJ-1 isoform volumes over total DJ-1 levels were normalized to the control values in each independent experiment. Means \pm SEM are shown. (C) Quantification of DJ-1 isoform 2 (DJ-1 isoform 2/total DJ-1) among control and disease whole blood samples; (D) Quantification of DJ-1 isoform 6 (DJ-1 isoform 6/total DJ-1) among control and disease whole blood samples. Asterisks denote data points representing an experimental group significantly different statistically $\left(* p<0.05, * * p<0.01,{ }^{* * *} p<0.001\right)$ from the control group.

protein become more prominent in subjects with neurodegenerative disorders $^{36}$. The MS-identified phosphorylation, 4-HNE, and various other oxidation modifications are expected to lower the proteins $\mathrm{pI}$, which would shift its 2D spot toward the more acidic end of the gel $^{29,37-40}$. However, it is likely that there are many other PTMs that remain to be identified and it is the net effect of all of these modifications taken together that ultimately determines an isoform's pI. Examples of additional potentially unidentified modifications that can influence $\mathrm{pI}$ include glycosylation, acetylation, deamidation, and glutathionylation $^{39,41}$. In fact, one of the major challenges of MS identification of peptides and proteins is that a significant portion of peptides cannot be recognized without a known mass shift. Additionally, the relative abundance of peptide sequences containing signature PTMs responsible for an isoform's $\mathrm{pI}$ shift may be too low to be detected without substantial enrichment of the specific modified form. Furthermore, biologically modified peptides may undergo a series of ex vivo modifications during sample preparation which compromise the integrity of the original peptide thus preventing its identification.

It has been demonstrated that dopamine treatment in human SHSY5Y neuroblastoma cells may cause a DJ-1 "isoform shift" toward more acidic $\mathrm{pIs}^{42}$, raising the possibility that HNE-modified DJ-1 in our study could be influenced by dopamine treatment of PD patients.
However, we were unable to observe such a shift of total levels of DJ-1 isoforms in human whole blood (Fig. 4); furthermore, the HNEmodified isoforms of interest reported here (\#4 and \#6) are localized on the more basic end of the DJ-1 pI range. We also incubated human whole blood with dopamine and did not observe the modifications of interest (Suppl. Fig. 4). It is therefore highly unlikely dopamine therapy in PD patients is capable of inducing the 4-HNE modified DJ-1 changes observed in this study.

In summary, we have confirmed that measurement of total DJ-1 in blood is not suitable for distinguishing $\mathrm{PD}$ or $\mathrm{AD}$ patients from age-matched controls. Furthermore, we identified 7 isoforms of DJ1 in whole blood samples, several of which contain newly described PTMs in addition to the previously characterized Cys-106 oxidation. The blood levels of two of these isoforms appeared to be significantly altered in both $\mathrm{PD}$ and $\mathrm{AD}$ patients as compared to controls. Most significantly, we have found that the ratio of the 4HNE-modified fraction of two of these isoforms increases with respect to both $\mathrm{PD}$ diagnosis and $\mathrm{PD}$ severity, a finding which was further confirmed in an independent population. These findings provide an indication of the diagnostic potential of DJ-1 isoforms in whole blood. These results, of course, need to be validated, ideally using ELISA or Luminex technology that can readily measure individual samples. 
A

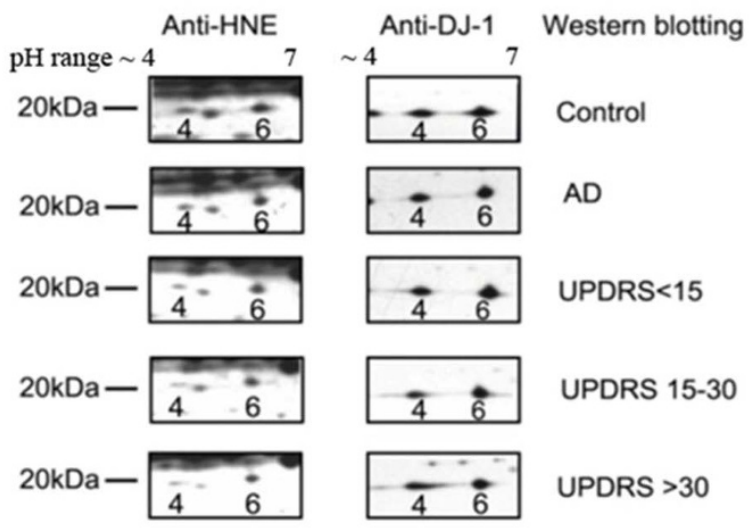

B

Fraction HNE isoform No.4

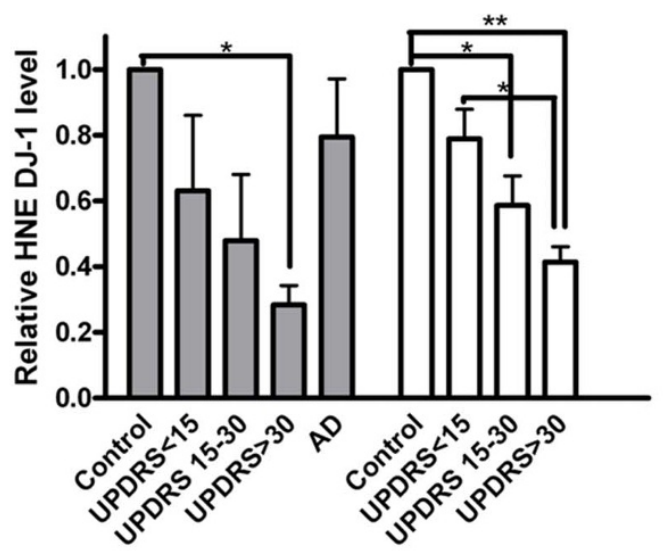

Fraction HNE isoform No.4 /Total HNE DJ-1

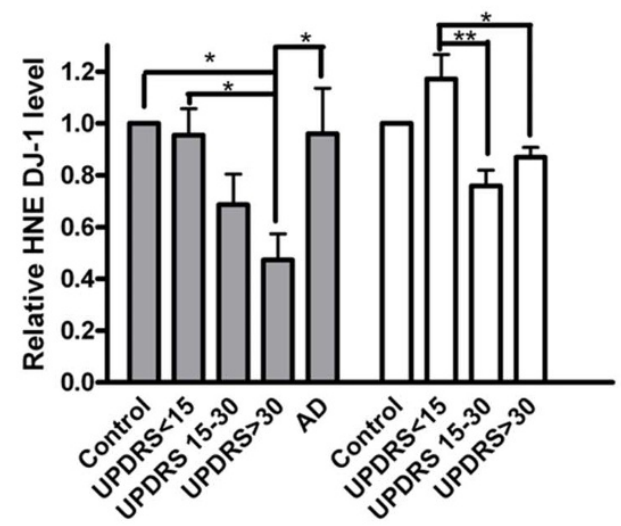

D

Fraction HNE isoform No.6

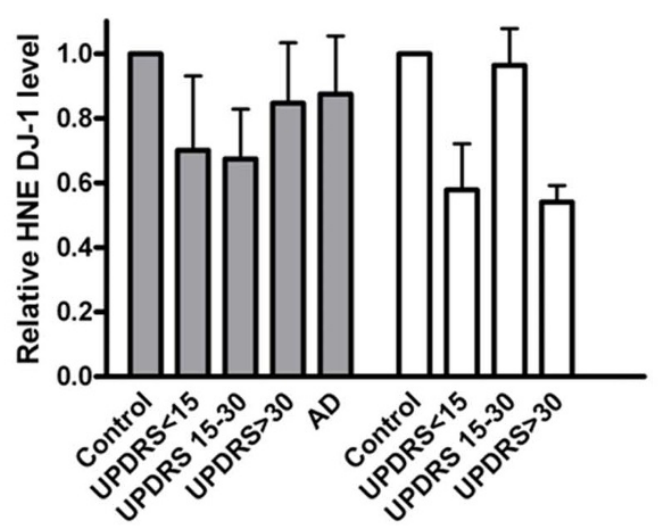

E

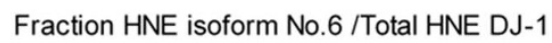

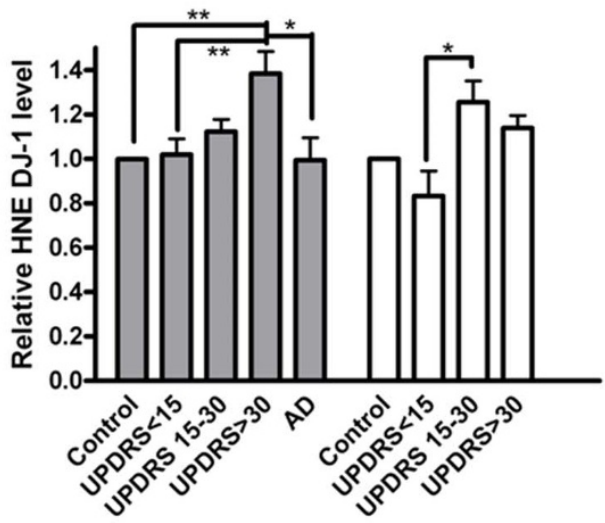

Figure 5 | The expression of HNE-altered DJ-1 isoforms was significantly different in whole blood samples obtained from AD patients, PD patients at various stages, and controls in discovery and validation cohorts. (A) 2-DE western blotting of HNE-modified DJ-1 isoforms in pooled whole blood samples. Left, blotting using an anti-HNE antibody; right, the same membrane was stripped and re-probed with an anti-DJ-1 antibody.

(B)-(E) Quantification of 2-DE western blotting of DJ-1 isoforms 4 and 6 in discovery (solid bars) and validation set (open bars): HNE-modified isoform 4 as a relative fraction (mean \pm SEM) of total isoform 4 (HNE-isoform 4/DJ-1 isoform 4 total) (B) and of total HNE-modified DJ-1 (HNE-isoform 4/ [HNE-isoform $4+6]$ ) (C), HNE-modified isoform 6 as a relative fraction (mean \pm SEM) of total isoform 6 (D) and of total HNE-modified DJ-1 (E); the values in each independent experiment were normalized to the values of the control group. $\left(*\right.$ indicates $p<0.05$; ${ }^{* *}$ indicates $\left.p<0.01\right)$. 


\section{Methods}

Participants. Subjects for both the discovery (119 total; 30 controls, 14 AD patients, 75 PD patients) and validation (114 total; 30 controls, 84 PD patients) studies were recruited from the Veterans Affairs Puget Sound Health Care System at Seattle and Washington University (St. Louis, MO). All participants provided informed consent and underwent evaluation by a neurologist that consisted of a structured interview, neurological examination, laboratory tests, and neuropsychological assessments. All control subjects were community volunteers who had Mini Mental State Exam Scores $>27$, paragraph recall scores $>6$, no history of neurological disease, and no history or evidence of cognitive or functional decline. The clinical diagnosis of probable AD was made using NINDS-ADRDA criteria ${ }^{13}$, applied at a clinical consensus conference in the Clinical Core of the University of Washington Alzheimer's Disease Research Center. All PD patients met UK PD Society Brain Bank clinical diagnostic criteria for $\mathrm{PD}^{14}$. PD patient samples were further categorized based on UPDRS Part III on-state motor scores to approximate disease stage. To correlate the performance of DJ-1 with PD severity, patients with UPDRS scores $<15$ were defined as having early-stage PD, those with scores ranging from 15-30 were classified as middle-stage PD, while those with scores $>30$ were classified as late-stage PD patients. Demographic data for all subjects and UPDRS scores for all PD patients used in the study are listed in Table 1 (discovery set) and Table 2 (validation set). The study was approved by the Institutional Review Boards of all participating institutions.

Whole blood sample preparation. After adding 10\% protease inhibiting cocktail, $130 \mu \mathrm{L}$ of whole blood from each individual sample were pooled according to Table 1 and Table 2. The pooled samples were lysed by adding an equal volume of lysis buffer [20 mM Tris (pH 7.5), $150 \mathrm{mM} \mathrm{NaCl}, 1$ mM EDTA, 1 mM EGTA, 1\% Triton X-100, $2.5 \mathrm{mM}$ sodium pyrophosphate $\left(\mathrm{Na}_{4} \mathrm{P}_{2} \mathrm{O}_{4}\right), 1 \mathrm{mM} \beta$-glycerophosphate, $1 \mathrm{mM}$ sodium orthovanadate $\left(\mathrm{Na}_{3} \mathrm{VO}_{4}\right)$ and $1 \mu \mathrm{g} / \mathrm{mL}$ leupeptin], vortexed for $2 \mathrm{~min}$, placed on ice for $5 \mathrm{~min}$, and vortexed again for $2 \mathrm{~min}$. After freezing at $-80^{\circ} \mathrm{C}$ for at least $30 \mathrm{~min}$, the samples were thawed in a $37^{\circ} \mathrm{C}$ water bath for $2-3 \mathrm{~min}$ and then vortexed for $2 \mathrm{~min}$ and centrifuged at $14,000 \times g$ for $10 \mathrm{~min}$ at $4^{\circ} \mathrm{C}$. The supernatant was then transferred to a new tube and either stored at $-80^{\circ} \mathrm{C}$ or immediately used.

Differential solubilization (DS) methods to enrich DJ-1. Low molecular weight proteins, including DJ-1, were enriched from whole blood according to a procedure described previously ${ }^{15,16}$, with the following slight modifications: $50 \mu \mathrm{L}$ whole blood samples were diluted with $100 \mu \mathrm{L}$ denaturating solution [7 M urea, $2 \mathrm{M}$ thiourea, and $20 \mathrm{mM}$ dithiothreitol (DTT)] and precipitated with $4.5 \mathrm{~mL}$ acetone while mixing at $4^{\circ} \mathrm{C}$ for $1 \mathrm{~h}$, then centrifuged at $19,000 \times g$ for $15 \mathrm{~min}$ at $4^{\circ} \mathrm{C}$. The pellet was resolved in $1 \mathrm{~mL} 70 \%$ acetonitrile (ACN) containing $12 \mathrm{mM} \mathrm{HCl}$ and mixed at $4{ }^{\circ} \mathrm{C}$ for $1 \mathrm{~h}$. After centrifuging at $19,000 \times g$ for $15 \mathrm{~min}$ at $4^{\circ} \mathrm{C}$ again, the supernatant was dried down using a CentriVap Concentrator (Labconco, Kansas City, MO, USA), resuspended in $200 \mu \mathrm{L} 2$-DE rehydration and lysis buffer [ $8 \mathrm{M}$ urea, $50 \mathrm{mM}$ DTT, $2 \%(\mathrm{w} / \mathrm{v})$ CHAPS, $0.2 \%(\mathrm{w} / \mathrm{v})$ carrier ampholytes, and $0.0002 \%$ bromophenol blue], and stored at $-80^{\circ} \mathrm{C}$ until use.

Immunoprecipitation. Immunoprecipitation (IP) was carried out using either Invitrogen M-280 Tosylactivated Dynabeads (Carlsbad, CA, USA) (for HNE DJ-1) or Santa Cruz ImmunoCruz IP/WB Optima F System (Santa Cruz, CA, USA) (for phosphorylated DJ-1) with slight modifications to the manufacturer's instructions. Briefly, for HNE DJ-1 detection, $40 \mu \mathrm{g}$ of dialyzed anti-DJ-1 antibody (Novus, Littleton, CO, USA) were coupled with $40 \mu \mathrm{L}$ Dynabeads and then incubated with $1 \mathrm{~mL}$ of lysed whole blood (diluted $1: 1$ in PBS) at $4{ }^{\circ} \mathrm{C}$ overnight. For phosphorylated DJ-1 detection, $1 \mathrm{~mL}$ matrixF was coupled to $100 \mu \mathrm{g}$ antibody and incubated with $4 \mathrm{~mL}$ whole blood. Following incubation, beads were washed 3 times with PBS and 1 time with Milli-Q water and then incubated in $100 \mu \mathrm{L}$ of 2-DE rehydration buffer for $2 \times 20 \mathrm{~min}$. The supernatants were then combined and used for subsequent $2-\mathrm{DE}$ western blotting analyses.

Two-dimensional electrophoresis. The 2-DEs were performed as described previously ${ }^{17}$. Briefly, for first-dimension electrophoresis, $2 \mu \mathrm{L}$ lysed blood or enriched samples were resolved in $200 \mu \mathrm{L}$ rehydration buffer and applied to an $11-\mathrm{cm} \mathrm{pH} \mathrm{4-7}$ ReadyStrip ${ }^{\mathrm{TM}}$ IPG strip (Bio-Rad, Hercules, CA, USA) for rehydration at room temperature for $20 \mathrm{~h}$, then isoelectric focusing (IEF) was performed using a PROTEAN ${ }^{\circledR}$ IEF cell (Bio-Rad) at 250-V linear ramp for $1 \mathrm{~h} ; 500-\mathrm{V}$ rapid ramp for $1 \mathrm{~h} ; 1000-\mathrm{V}$ rapid ramp for $1.5 \mathrm{~h} ; 8000-\mathrm{V}$ linear ramp for $5 \mathrm{~h} ; 8000-\mathrm{V}$ rapid ramp for a total of 35,000 volt-hours; and 500-V rapid ramp as a holding step for additional volt-hours (typical total volt-hours are $\sim 62,000$ ). All the processes above were carried out at $20^{\circ} \mathrm{C}$ with a current limit of $50 \mu \mathrm{A}$ per IPG strip.

For second-dimension electrophoresis, IPG strips were equilibrated for $10 \mathrm{~min}$ in buffer I [ $37.5 \mathrm{mM}$ Tris- $\mathrm{HCl}$ ( $\mathrm{pH} 8.8$ ) containing $6 \mathrm{M}$ urea, $2 \% \mathrm{w} / \mathrm{v}$ SDS, $20 \% \mathrm{v} / \mathrm{v}$ glycerol, and 2\% DTT] and then re-equilibrated for $10 \mathrm{~min}$ in buffer II [same buffer containing $2.5 \%$ iodoacetamide in place of DTT]. For the HNE immunoblotting process, IPG strips were soaked in $0.1 \mathrm{M}$ Tris $\mathrm{HCl}(\mathrm{pH} 8.5)$ containing $0.86 \mathrm{mM}$ EDTA and $100 \mathrm{mM} \mathrm{NaBH}_{4}$ (Sigma-Aldrich, St. Louis, MO, USA) for $15 \mathrm{~min}$ then washed in the same buffer without $\mathrm{NaBH}_{4}$ for 15 min before equilibration with DTT and iodoacetamide. Second-dimension electrophoresis (SDS-PAGE) was performed using 10-20\% Precast Criterion Tris- $\mathrm{HCl}$ linear gels (Bio-Rad) with a constant voltage of $120 \mathrm{~V}$ for $10 \mathrm{~min}$ then $160 \mathrm{~V}$ for $70 \mathrm{~min}$ until the dye front reached the bottom of the gels. Silver staining was used to visualize 2-DE spots to be excised.
Western blotting and quantification. Western blotting on 2-DE gels was performed as previously described ${ }^{18}$. Briefly, gels containing samples were transferred to a PVDF membrane for $2 \mathrm{~h}$ at $800 \mathrm{~mA}$ in transfer buffer [ $48 \mathrm{mM}$ Tris, $39 \mathrm{mM}$ glycine, and $15 \%$ methanol] at $4{ }^{\circ} \mathrm{C}$. For HNE-modified protein detection, membranes were blocked in Tris buffered saline ( $\mathrm{pH} 8.0)$ containing $0.1 \%(\mathrm{v} / \mathrm{v})$ Tween (TBS-T) and $5 \%$ $(\mathrm{w} / \mathrm{v})$ bovine serum albumin (BSA) at room temperature for $1 \mathrm{~h}$ and then incubated overnight at $4{ }^{\circ} \mathrm{C}$ with a rabbit anti-reduced HNE antibody ${ }^{15}$ at a dilution of $1: 1000$. After washing with TBS-T, the membranes were incubated with horseradish peroxidase-conjugated goat anti-rabbit antibody at a dilution of $1: 20,000$ in TBS-T containing $3 \%(\mathrm{w} / \mathrm{v})$ BSA at room temperature for $1 \mathrm{~h}$. The immunostained proteins were detected using ECL Plus (GE Healthcare, Buckinghamshire, UK) and exposed for $2 \mathrm{~min}$. The developed films were scanned using a Versa ${ }^{\mathrm{TM}}$ DOC Image System Model 3000 and analyzed with PDQEST 7.0 software (Bio-Rad, Hercules, CA, USA)

For phosphorylated threonine protein detection, membranes were blocked in $5 \%$ (w/v) BSA/TBS-T and then washed twice with TBS-T and once with TBS. The membrane was then incubated with the mouse anti-Phospho-Threonine antibody Q7 (Qiagen, Valencia, CA, USA) (diluted $1: 100$ in TBS-T) overnight at $4^{\circ} \mathrm{C}$, followed by rabbit anti-mouse secondary antibody (diluted $1: 20,000$ in $10 \%$ non-fat milk/TBS-T) for $1 \mathrm{~h}$ at room temperature. For total DJ-1 western blotting, the blocking buffer was $5 \%(\mathrm{w} / \mathrm{v})$ non-fat milk in TBS-T, the primary antibody was a rabbit anti-DJ-1 antibody (Novus) diluted $1: 2000$, and the secondary antibody and dilution were the same as described above. The PVDF membranes were stained by Coomassie R-350 to verify equal loading.

Mass spectrometry. Spots identified using DS-enriched methods were excised from sliver stained 2-D gels according to the 2-DE western blotting result, digested with trypsin, and analyzed by LTQ-Orbitrap MS as previously described ${ }^{19}$. Briefly, target spots were excised and placed in a 1.5 -mL plastic microcentrifuge tube, washed with MilliQ water three times, followed by $50 \%$ ACN several times, and dried in a vacuum centrifuge. The gel pieces were digested with $20 \mu \mathrm{L}$ of $20 \mathrm{ng} / \mu \mathrm{L}$ sequencing grade trypsin (Promega, San Luis Obispo,CA,USA) in $50 \mathrm{mM}$ ammonium bicarbonate ( $\mathrm{pH}$ $8.0)$ for $16 \mathrm{~h}$ at $37^{\circ} \mathrm{C}$. The peptides were then extracted once in $30 \mu \mathrm{L}$ of $50 \mathrm{mM}$ ammonium bicarbonate and twice in $30 \mu \mathrm{L}$ extraction buffer [ $50 \%(\mathrm{v} / \mathrm{v}) \mathrm{ACN}$ and $5 \%$ $(\mathrm{v} / \mathrm{v})$ formic acid], with $10 \mathrm{~min}$ incubation and occasional gentle vortex mixing each time. The supernatants saved from each extraction were combined, dried down, and reconstituted in buffer containing $5 \% \mathrm{ACN}$ and $0.1 \%$ formic acid.

The protein identification was performed using a LTQ-Orbitrap hybrid MS (Thermo Scientific, San Jose, CA, USA) with the following conditions: Peptides were separated online with $0.75 \mu \mathrm{m}$ i.d. $\times 20 \mathrm{~cm}$ home-packed columns $(100 \AA$ Magic C18AQ: Michrom Bioresources, Auburn, CA, USA) with a 60 min 5-35\% ACN/ water gradient containing $0.1 \%$ formic acid using a Nano-Acquity UPLC (Waters, Milford, MA, USA). The electrospray voltage was applied via a liquid microtee junction located in between the precolumn and analytical column. All ions were measured in positive mode and automatic gain control was optimized to maintain constant ion populations. After data dependent acquisition, tandem MS data was searched by SEQUEST (v27) against the International Protein Index human protein database (v3.68) and protein identifications were filtered using PeptideProphet. All methionines were allowed to be oxidized and cysteine residues were considered as being alkylated by iodoacetamide and were assigned a mass of $160 \mathrm{Da}$. The lysine, histine, and cysteine resides were set as being 4-HNE modified with a mass of +156 Da. The threonine, tyrosine, and serine residues were set as being phosphorylated with a mass of $+80 \mathrm{Da}$. The cysteine residues also were set as being oxidized to cysteic acid with a mass of $+48 \mathrm{Da}$.

Statistical analysis. All the western blotting data were collected from at least three independent experiments and analyzed using Prism 4.0 (Graphpad, San Diego, CA, USA). Group differences were assessed using one-way analysis of variance (ANOVA) followed by the post-hoc Tukey honestly significant difference test. Asterisks denote data points representing an experimental group significantly different statistically $(p<0.05)$ from the control group.

1. Lang, A. E. \& Lozano, A. M. Parkinson's disease. Second of two parts. NEngl J Med 339, 1130-1143 (1998).

2. Wright Willis, A., Evanoff, B. A., Lian, M., Criswell, S. R. \& Racette, B. A. Geographic and ethnic variation in Parkinson disease: a population-based study of US Medicare beneficiaries. Neuroepidemiology 34, 143-151 (2010).

3. Jankovic, J. Parkinson's disease: clinical features and diagnosis. J Neurol Neurosurg Psychiatry 79, 368-376 (2008).

4. Tolosa, E., Wenning, G. \& Poewe, W. The diagnosis of Parkinson's disease. Lancet Neurol 5, 75-86 (2006).

5. Hong, Z. et al. DJ-1 and alpha-synuclein in human cerebrospinal fluid as biomarkers of Parkinson's disease. Brain 133, 713-726 (2010).

6. Bonifati, V. et al. DJ-1( PARK7), a novel gene for autosomal recessive, early onset parkinsonism. Neurol Sci 24, 159-160 (2003).

7. Taira, T. et al. DJ-1 has a role in antioxidative stress to prevent cell death. EMBO Rep 5, 213-218 (2004).

8. Diamandis, E. P., Yousef, G. M., Petraki, C. \& Soosaipillai, A. R. Human kallikrein 6 as a biomarker of Alzheimer's disease. Clin Biochem 33, 663-667 (2000).

9. Bogdanov, M. et al. Metabolomic profiling to develop blood biomarkers for Parkinson's disease. Brain 131, 389-396 (2008). 
10. Shehadeh, L. A. et al. SRRM2, a potential blood biomarker revealing high alternative splicing in Parkinson's disease. PLoS One 5, e9104 (2010).

11. Stern, E. et al. Label-free biomarker detection from whole blood. Nat Nanotechnol 5, 138-142 (2010).

12. Shi, M. et al. Significance and confounders of peripheral DJ-1 and alpha-synuclein in Parkinson's disease. Neurosci Lett 480, 78-82 (2010).

13. McKhann, G. et al. Clinical diagnosis of Alzheimer's disease: report of the NINCDS-ADRDA Work Group under the auspices of Department of Health and Human Services Task Force on Alzheimer's Disease. Neurology 34, 939-944 (1984).

14. Gibb, W. R. \& Lees, A. J. The relevance of the Lewy body to the pathogenesis of idiopathic Parkinson's disease. J Neurol Neurosurg Psychiatry 51, 745-752 (1988).

15. Montine, K. S. et al. Distribution of reducible 4-hydroxynonenal adduct immunoreactivity in Alzheimer disease is associated with APOE genotype. I Neuropathol Exp Neurol 57, 415-425 (1998).

16. Kawashima, Y. et al. High-yield peptide-extraction method for the discovery of subnanomolar biomarkers from small serum samples. J Proteome Res $\mathbf{9}$, 1694-1705 (2010)

17. Shi, M. et al. Mortalin: a protein associated with progression of Parkinson disease? I Neuropathol Exp Neurol 67, 117-124 (2008).

18. Jin, J. et al. Quantitative proteomic analysis of mitochondrial proteins: relevance to Lewy body formation and Parkinson's disease. Brain Res Mol Brain Res 134, 119-138 (2005).

19. Devic, I. et al. Salivary alpha-synuclein and DJ-1: potential biomarkers for Parkinson's disease. Brain 134, el78 (2011)

20. Gygi, S. P., Corthals, G. L., Zhang, Y., Rochon, Y. \& Aebersold, R. Evaluation of two-dimensional gel electrophoresis-based proteome analysis technology. Proc Natl Acad Sci USA 97, 9390-9395 (2000).

21. Waragai, M. et al. $\alpha$-Synuclein and DJ-1 as Potential Biological Fluid Biomarkers for Parkinson's Disease. Int J Mol Sci 11, 4257-4266 (2010).

22. Waragai, M. et al. Plasma levels of DJ-1 as a possible marker for progression of sporadic Parkinson's disease. Neurosci Lett 425, 18-22 (2007).

23. Maita, C. et al. Secretion of DJ-1 into the serum of patients with Parkinson's disease. Neurosci Lett 431, 86-89 (2008).

24. Wang, J., Hoekstra, J. G., Zuo, C., Cook, T. J. \& Zhang, J. Biomarkers of Parkinson's disease: current status and future perspectives. Drug Discovery Today (2012), in press: http://dx.doi.org/10.1016/j.drudis.2012.09.001

25. Rush, J. et al. Immunoaffinity profiling of tyrosine phosphorylation in cancer cells. Nat Biotechnol 23, 94-101 (2005).

26. Choi, J. et al. Oxidative damage of DJ-1 is linked to sporadic Parkinson and Alzheimer diseases. J Biol Chem 281, 10816-10824 (2006).

27. Saito, Y. et al. Preparation and application of monoclonal antibodies against oxidized DJ-1. Significant elevation of oxidized DJ-1 in erythrocytes of early-stage Parkinson disease patients. Neurosci Lett 465, 1-5 (2009).

28. Kinumi, T., Kimata, J., Taira, T., Ariga, H. \& Niki, E. Cysteine-106 of DJ-1 is the most sensitive cysteine residue to hydrogen peroxide-mediated oxidation in vivo in human umbilical vein endothelial cells. Biochem Biophys Res Commun 317, 722-728 (2004)

29. Canet-Avilés, R. M. et al. The Parkinson's disease protein DJ-1 is neuroprotective due to cysteine-sulfinic acid-driven mitochondrial localization. Proc Natl Acad Sci USA 101, 9103-9108 (2004).

30. Hao, L. Y., Giasson, B. I. \& Bonini, N. M. DJ-1 is critical for mitochondrial function and rescues PINK1 loss of function. Proc Natl Acad Sci USA 107, 9747-9752 (2010).

31. Tsuboi, Y. et al. DJ-1, a causative gene product of a familial form of Parkinson's disease, is secreted through microdomains. FEBS Lett 582, 2643-2649 (2008).

32. Akazawa, Y. O. et al. Elevation of oxidized DJ-1 in the brain and erythrocytes of Parkinson disease model animals. Neurosci Lett 483, 201-205 (2010).
33. Yoritaka, A. et al. Immunohistochemical detection of 4-hydroxynonenal protein adducts in Parkinson disease. Proc Natl Acad Sci USA 93, 2696-2701 (1996).

34. Selley, M. L. (E)-4-hydroxy-2-nonenal may be involved in the pathogenesis of Parkinson's disease. Free Radic Biol Med 25, 169-174 (1998).

35. Ishimura, A. et al. Comparative study of hydrogen peroxide- and 4-hydroxy-2nonenal-induced cell death in HT22 cells. Neurochem Int 52, 776-785 (2008).

36. Natale, M. et al. A meta-analysis of two-dimensional electrophoresis pattern of the Parkinson's disease-related protein DJ-1. Bioinformatics 26, 946-952 (2010).

37. Baglo, Y. et al. Photodynamic therapy with hexyl aminolevulinate induces carbonylation, posttranslational modifications and changed expression of proteins in cell survival and cell death pathways. Photochem Photobiol Sci 10, 1137-1145 (2011).

38. Duan, X., Kelsen, S. G. \& Merali, S. Proteomic analysis of oxidative stressresponsive proteins in human pneumocytes: insight into the regulation of DJ-1 expression. J Proteome Res 7, 4955-4961 (2008).

39. Ferrington, D. A. \& Kapphahn, R. J. Catalytic site-specific inhibition of the $20 \mathrm{~S}$ proteasome by 4-hydroxynonenal. FEBS Lett 578, 217-223 (2004).

40. Garcia, A. et al. Differential proteome analysis of TRAP-activated platelets: involvement of DOK-2 and phosphorylation of RGS proteins. Blood 103, 2088-2095 (2004).

41. Ethen, C. M., Reilly, C., Feng, X., Olsen, T. W. \& Ferrington, D. A. Age-related macular degeneration and retinal protein modification by 4-hydroxy-2-nonenal. Invest Ophthalmol Vis Sci 48, 3469-3479 (2007).

42. Alberio, T. et al. Changes in the two-dimensional electrophoresis pattern of the Parkinson's disease related protein DJ-1 in human SH-SY5Y neuroblastoma cells after dopamine treatment. IUBMB Life 62, 688-692 (2010).

\section{Acknowledgments}

The authors deeply appreciate all participants' donation of time and blood for making our study possible. We would also like to thank John D. Chapman for providing technical expertise and assistance in LTQ-Orbitrap MS proteomic processing of our samples. This work was supported by the National Institutes of Health [grant numbers NS057567, NS065070, P50NS062684, NS060252, AG025327, AG033398, ES004696, ES016873, ES019277, P30ES007033, ES015459-02]; Department of Veterans Affairs [grant number 1I01BX000531]; and Shaw endowment.

\section{Author contributions}

J.Z. conceived and supervised the project. X.L. performed all immunoblotting studies and MS/MS sample preparation and data analysis. T.J.C., X.L., M.S. and J.Z. drafted the manuscript, and all listed authors contributed to further revisions. C.P.Z., J.B.L., E.R.P., S.C.H., B.A.R. and H.C. were responsible for patient characterization and sample collection. T.J.M. contributed experimental design for 4-HNE identification and quantification. K.C.C. contributed experimental pooling design and statistical expertise. C.P., J.S.E. and D.R.G. performed mass spectrometry analyses. All authors critically reviewed the manuscript.

\section{Additional information}

Supplementary information accompanies this paper at http://www.nature.com/ scientificreports

Competing financial interests: The authors declare no competing financial interests.

License: This work is licensed under a Creative Commons

Attribution-NonCommercial-NoDerivs 3.0 Unported License. To view a copy of this license, visit http://creativecommons.org/licenses/by-nc-nd/3.0/

How to cite this article: Lin, X. et al. DJ-1 isoforms in whole blood as potential biomarkers of Parkinson disease. Sci. Rep. 2, 954; DOI:10.1038/srep00954 (2012). 